\title{
Cibler les inégalités : Contribution de l'approche des Capabilités aux études de la population.
}

\author{
Claudine Sauvain-Dugerdil \\ Institut d'études démographiques et du parcours de vie (I-DEMO), Université de Genève, \\ Claudine.Sauvain@unige.ch
}

\section{Résumé}

Cet article présente les objectifs du projet qui est l'objet de ce numéro spécial, à savoir l'application de l'approche des Capabilités (AC) aux études démographiques pour mieux comprendre les disparités croissantes de développement humain et sélectionner les meilleurs indicateurs pour identifier les inégalités d'opportunités. Nous présentons ici l'apport de l'AC pour les études démographiques, puis les caractéristiques des deux pays ouest-africains considérés Mali et Ghana - et, finalement, les sujets traités. L'application de l'AC ubstitue l'approche classique des déterminants de résultats atteints par une centration sur l'éventail d'opportunités et les disparités. Les huit articles qui suivent abordent trois domaines centraux en démographie sociale, particulièrement en Afrique de l'Ouest, et pour lesquels de riches données existent: la santé, à travers les facteurs de risques et la montée de l'obésité; l'intégration sociale exprimée par les activités professionnelles et la participation sociale; le planning familial analysé à travers la contraception et l'espacement des naissances.

Mots-clés: Approche de Capabilités, Mali, Ghana, population et développement.

\section{Introduction: Cibler les inégalités}

Ce projet de recherché est né du constat de deux paradoxes dans le champ population et développement. D'une part, on observe des avancées importantes dans l'atteinte de plusieurs objectifs démographiques et sanitaires au niveau planétaire, parallèlement à des disparités croissantes entre et dans les populations (Bhutta and Black, 20I3). D'autre part, les analystes sont submergés par la masse de nouvelles données mais, régulièrement, des mesures clés font défaut par absence de cadre théorique ou simplement de compréhension de l'interaction entre les processus sociaux et la croissance économique. Les articles qui suivent examinent ces deux questions en appliquant l'approche des Capabilités, un cadre peu commun dans les études de population, qui adresse simultanément ces deux problèmes.

$>$ Des progrès qui engendrent la croissance des inégalités
Les rapports sur les Objectifs du Millénaire pour le développement (OMD) (ONU, 2013) montrent que plusieurs objectifs risquent de ne pas être atteints et que les progrès sont très inégaux entre les régions et les pays, mais aussi au sein des pays. Ceci est démontré dans de nombreux cas, mais particulièrement en ce qui concerne l'égalité de genre. Par exemple, en Afrique du Nord l'amélioration globale de la survie de l'enfant semble bénéficier davantage aux garçons, comme le montre la moindre baisse de la mortalité des filles par rapport à celle des garçons (Tabutin et al., 2007). On reconnaît de plus en plus que les progrès peuvent bénéficier plus au mieux lotis et que, par conséquent, les mesures moyennes des résultats peuvent masquer des inégalités croissantes (Waage et al., 2010). Cette association paradoxale entre progrès et inégalités, reconnue historiquement en Europe pendant la période de croissance de la fin du XIXe siècle (Woods, 2003; Woods, 2000), est 
dramatiquement illustrée dans le monde contemporain par le cas de l'Inde (Drèze et Sen, 2013). Des publications récentes redécouvrent d'anciennes évidences montrant que les innovations ne sont souvent accessibles qu'à une petite minoritéii. En outre, il est de plus en plus reconnu que les inégalités et l'exclusion sociale sont des problèmes persistants qui progressent même dans les pays dits développés (CEE-ONU, rapport régional CIPD au-delà de 20/4iii). Cette prise de conscience doit beaucoup aux analyses de Wilkinson et Pickett (2009) qui montrent que les sociétés ayant une répartition plus équitable du revenu ont des populations en meilleure santé et moins de problèmes sociaux. Ces auteurs mettent en évidence les effets pervers des inégalités sur la santé physique et mentale, l'abus de drogues, l'éducation, la délinquance, l'obésité, la mobilité sociale, la confiance et la vie communautaire, la violence, les grossesses d'adolescentes et le bien-être de l'enfant.

Comme l'ont souligné Drèze et Sen (20l3), la leçon à tirer du cas de l'Inde est que la croissance économique devrait s'accompagner d'un développement humain, c'est-à-dire d'un renforcement des Capabilités humaines. C'est la même notion que réaffirme le renouvellement des engagements relatifs au paradigme de la Conférence internationale sur la Population et le développement (CIPD) qui encourage à " investir dans le développement des personnes, en particulier des femmes et des groupes vulnérables, afin que les hommes et les femmes soient en mesure de prendre des décisions et faire des choix qui leur permettent d'atteindre un plus grand bien-être "(CEE-ONU au-delà de 20I4).

\section{$>$ Une pléthore de données mais des mesures lacunaires}

Dans le but légitime de mesurer les progrès de façon tangible plutôt que d'en rester à des souhaits vertueux, le cadre des OMD a défini une série d'objectifs quantitatifs. Bien que louable, il s'est avéré problématique au moins pour deux raisons.
D’une part, il a généré une industrie impressionnante de récolte de données par les gouvernements, les organisations non gouvernementales et la société civile. Aux riches bases de données standardisées initiées avec l'enquête mondiale sur la fécondité, puis les enquêtes démographiques et de santé (EDS), se sont ajoutées des études menées par d'autres agences onusiennes (enquêtes sur le niveau de vie de la Banque mondiale et l'enquête d'indicateurs multiples (MICS) de I'UNICEF). Ces données sont largement utilisées pour quantifier les progrès vers les OMD et les résultats des programmes, ainsi que pour fixer les priorités des actions en matière de population et de services de santé reproductive et sexuelle. Les efforts internationaux pour fournir des preuves des évolutions démographiques, sociales et sanitaires, en particulier dans les pays pauvres et peu documentés, ont aussi mis en évidence des caractéristiques des transitions démographiques et sanitaires différentes de ce qui avait été observé en Europe et en Amérique du Nord. Néanmoins, comme en témoignent les cas du Mali et du Ghana, les deux pays considérés ici, cette explosion de collecte de données prise en charge par des organismes étrangers répond essentiellement à leur préoccupation de mesurer les progrès accomplis dans les indicateurs les plus importants pour leurs propres programmes. Ainsi un système parallèle s'est développé, souvent hors du contrôle de l'institution nationale chargée de la collecte de données.

D'autre part, ces données sont généralement utilisées pour produire des indicateurs de résultats moyens dans certains secteurs ou sous-secteurs, dans le but de comparer les réalisations des pays dans des domaines spécifiques. Dans la plupart des cas, les renseignements recueillis ne répondent pas aux besoins de planification au niveau local et les données sont sous-utilisées dans les pays euxmêmes. Clairement, les observations des tendances des niveaux de fécondité, de scolarisation, de mortalité infantile et d'autres 
mesures de développement social ne fournissent pas d'indications sur les voies les plus efficaces pour réduire les inégalités. Même des cibles aussi spécifiques que le taux de scolarisation ou la prévalence contraceptive peuvent alors ne pas atteindre un but plus large d'amélioration de l'éducation ou d'accès des femmes à la possibilité de planifier leur famille (Yamin et Boulanger, 20l3).

Le défi du présent projet est de montrer que malgré leurs limites, les données recueillies pourraient être ré-analysées et ré-interprétées pour aider à comprendre les sources d'inégalités ou, comme souligné par Waage et al (2010), les besoins essentiels de développement humain.

\section{L'approche des Capabilités comme outil pour explorer les racines des inégalités d'opportunités.}

Ce projet de recherche applique l'approche des Capabilités (AC) dans les études démographiques afin de mieux comprendre les déterminants profonds, sous-jacents aux différences de comportements démographiques et les aspects associés de développement humain. En mettant l'accent sur la "liberté d'atteindre ", l'AC considère les multiples dimensions de la pauvreté, les causes des inégalités de bien-être, les privations réelles des gens, ainsi que leurs différents besoins. Elle met l'accent sur l'équité des opportunités plutôt que sur l'aboutissement en termes d'égalité, de satisfaction et d'insatisfaction. L'AC a été conçue comme une théorie dépassant les approches économiques basées sur les mesures du revenu, des indices de capital humain, d'utilité ou la possession de biens de base (voir article précédent). Elle se centre sur la situation réelle des personnes, sur les raisons pour lesquelles elles ont une capacité inégale à utiliser les ressources théoriquement à leur disposition. Le développement humain est donc considéré comme un "processus d'expansion des libertés réelles dont jouissent les personnes "; en d'autres termes, les Capabilités qui les rendent capables « de vivre la vie qu'ils ont raison de valoriser»(Sen, 1999).

Comme rappelé dans l'article précédant, la philosophie du Programme d'Action de la CIPD, le dénommé paradigme du Caire, partage les principes fondamentaux de l'AC. Ces derniers rencontrent les courants actuels de pensée en démographie sociale qui prennent en compte les inégalités, les intentions et les processus de prise de décision. Le contexte d'individualisation des comportements est au cœur de la théorie de la Deuxième Transition démographique (Van De Kaa, 1994; Lesthaeghe, 1995). Des analyses raffinés des différences individuelles doivent beaucoup au développement d'outils analytiques pour étudier le parcours de vie et à leur élargissement pour situer les individus dans leur entourage (Bonvalet et Lelièvre, dir, 2012 ; Basu, 2013), ou pour distinguer différents niveaux de déterminants en appliquant des analyses multi-niveaux (Courgeau, 2007), tandis que les méthodes mixtes ont pour but de prendre en compte les points de vue variés des personnes elles-mêmes. Des modélisations des intentions et des prises de décisions sont considérés en appliquant à la démographie des approches fondées sur l'action de l'individu (Billari et Fürnkranz-Prskawetz, 2003) et en créant des ponts vers les théories développées en psychologie ou sociologie, comme la théorie du comportement planifié (Ajzen 1988; Billari et al., 2005), ou de la fonction de production sociale (Lindenberg, 1996).

L'AC n'offre pas de nouveaux outils, mais fournit un cadre avec des concepts organisateurs qui mettent l'accent sur le processus et pas simplement les résultats. En se centrant sur les possibilités réelles des gens, leur capacité d'agir (agency en anglais) - ce qui leur permet d'étendre leur éventail de possibilités - devient un enjeu central dans l'analyse des inégalités de bien-être. Nous considérons ici la capacité individuelle d'action directement en prenant en compte le point de vue de la personne dans les enquêtes qualitatives et indirectement comme une 
dimension transversale introduite par l'examen des disparités à deux niveaux :

I) Disparités individuelles dans l'accès et l'utilisation des ressources, observées en distinguant les inégalités de disponibilité et de qualité des ressources du contexte (y compris les services) des inégalités de ressources personnelles qui restreignent ou améliorent la capacité individuelle d'y accéder et de les utiliser.

2) Disparités dans la façon dont les individus font face aux aléas de la vie, ainsi que dans leurs motivations et leurs objectifs par l'examen du duo Capabilités-réalisations, c'est-à-dire l'écart entre les possibilités - en d'autres termes, la liberté de réaliser - et le résultat atteint. En particulier, comment les gens fonctionnent dans des situations de grande incertitude et comment ils gèrent les cadres institutionnels aux normes contraignantes?

Ainsi, l'action de l'individu n'est pas vue comme l'acte d'une personne déconnectée de son contexte mais suivant Emirbayer et Mische (1998), comme un "processus d'engagement social inscrit dans le temps" ou comme des projets " situés " (Bassand et al., 1975).

\section{Ghana et Mali: deux pays dans la même sous-région, mais avec des dynamiques démographiques et socio- économiques distinctes}

Le projet se situe dans deux pays d'Afrique occidentale, essentiellement parce que les chercheurs principaux $y$ ont une longue expérience de recherche sur les questions de population et de santé. L'Afrique de l'Ouest semble aussi particulièrement appropriée en raison d'un syncrétisme complexe entre des anciennes traditions et des ancrages socioculturels autochtones et la diffusion rapide de la modernisation et de la mondialisation. Bien que situés dans la même zone géographique et partageant de nombreuses caractéristiques socioculturelles, telles que des anciennes traditions d'espacement des naissances, le Ghana et le Mali proposent deux exemples distincts par leurs histoires coloniales. Des rythmes différents d'évolutions socioéconomiques récentes les placent aux extrémités du spectre de développement de la sous-région (tableau I).

Les deux pays ont connu un allongement significatif de l'espérance de vie, notamment par une baisse de la mortalité infantile. Celle-ci demeure cependant élevée et, au Mali, il y a eu peu de progrès ces dernières années. Le Mali reste encore beaucoup plus rural et les progrès socio-économiques sont marqués de fortes inégalités. Le retard est particulièrement élevé pour la scolarisation et le revenu par habitant. En 2012, le Mali est située au I82è rang sur 187 dans I'Indice de développement humain, alors que le Ghana, classé 135, est en tête de la sousrégion. L'indice de pauvreté multidimensionnelle (MPI iv) est quatre fois plus élevé au Mali qu'au Ghana (56 \% contre $14 \%$, Rapport sur le développement humain, 2013). Les deux pays sont caractérisés par une prévalence contraceptive faible malgré l'adoption de politiques de la famille, il y a plus de quarante ans au Ghana (1969) et plus de vingt ans au Mali (1991). Les dernières EDS montrent qu'au Mali, le taux de prévalence contraceptive est toujours inférieur à $10 \%$ et, au Ghana, elle stagne à un peu plus de $20 \%$ depuis deux décennies. La dynamique démographique dans les deux pays est également très différente. Alors que tous deux avaient une fécondité de près de sept enfants jusque vers 1975, elle a diminué considérablement au Ghana pour atteindre moins de 5 enfants vers 2000, alors qu'au Mali, le taux est resté à 6,5 (tableau I). Dans les deux pays, la fécondité a baissé considérablement dans la capitale, mais à des degrés divers : n'étant plus que de 2,5 dans le Grand Accra (DHS, 2008) mais restant à 4,8 naissances à Bamako (DHS, 2006). 
Tableau I. Dynamiques démographiques et socio-économiques au Ghana et au Mali.

\begin{tabular}{|c|c|c|c|c|}
\hline & $1950-55$ & $1980-85 / 1980$ & $2000-05 / 2000$ & $2005-10 / 2012$ \\
\hline \multicolumn{5}{|l|}{ GHANA } \\
\hline *Taux de fécondité (naiss vivantes par femme) & 6,4 & 6,4 & 4,6 & 4,2 \\
\hline * Taux fécondité adlescnetes (naiss pr 1000 femmes $15-19$ ans) & 149,0 & 128,3 & 77,0 & 67,6 \\
\hline *Taux de mortalité infantile (pr 1000 naiss vivantes) & 147 & 93 & 61 & 55 \\
\hline *Espérance de vie à la naissance (années) & 42,2 & 53,0 & 57,5 & 60,0 \\
\hline **Pourcentage urbain & 15,4 & 31,2 & 44 & 51,2 \\
\hline ***Indicateur de développe,enthumain (HDI) valeur & & 0,391 & 0,461 & 0,558 \\
\hline ***Nb moyen d'années scolarisation (adultes) & & 3,6 & 6,3 & 7 \\
\hline ***PNB par habitant en parité de pouvoir d'achat (PPA) & & 984 & $1^{\prime} 037$ & $1^{\prime} 684$ \\
\hline \multicolumn{5}{|l|}{ MALI } \\
\hline *Taux de fécondité (naiss vivantes par femme) & 6,5 & 7,1 & 6,8 & 6,8 \\
\hline * Taux fécondité adlescnetes (naiss pr 1000 femmes $15-19$ ans) & 187,7 & 189,89 & 185,1 & 180,9 \\
\hline *Taux de mortalité infantile (pr 1000 naiss vivantes) & 242 & 151 & 109 & 100 \\
\hline *Espérance de vie à la naissance (années) & 27,0 & 41,6 & 50,3 & 52,7 \\
\hline **Pourcentage urbain & 8,5 & 18,5 & 28,1 & 34,3 \\
\hline ***Indicateur de développe,enthumain (HDI) valeur & & 0,176 & 0,27 & 0,344 \\
\hline ***Nb moyen d'années scolarisation (adultes) & & 0,4 & 1,2 & 2 \\
\hline ***PNB par habitant en parité de pouvoir d'achat (PPA) & & 737 & 744 & 853 \\
\hline \multicolumn{5}{|l|}{ Sources : } \\
\hline \multicolumn{5}{|c|}{ *World Population Prospect, Population Division, United Nations, 2012, http://esa.un.org/undp/wpp/. } \\
\hline **World Urbanization Prospect, 2011, http://esa.un.org/undp/wup/ & & & & \\
\hline
\end{tabular}

Surtout, les deux pays diffèrent fortement en ce qui concerne la situation des femmes, telle qu'exprimée dans les profils de nuptialité et les opinions sur les questions de genre (Locoh, 2013). Le mariage précoce reste la règle au Mali alors qu'il a considérablement diminué au Ghana: au Mali, 7I \% des femmes sont mariées avant 18 ans (EDS, 2006), mais seulement $25 \%$ au Ghana (DHS, 2008). L'écart d'âge entre conjoints est resté stable depuis les années 1990, environ 6 ans au Ghana et 9 ans en milieu rural malien, tandis que, dans les villes maliennes, il a passé à 12 ans à cause du report du mariage des hommes. La proportion de femmes dans les unions polygames a considérablement diminué au Ghana, beaucoup moins au Mali, surtout en zone rurale : dans I'EDS 2008 au Ghana, 8I \% des femmes de 1549 ans se déclarent monogame, $93 \%$ dans le Grand Accra et $95 \%$ parmi celles avec un niveau de scolarisation du secondaire ou plus, au Mali, le même indicateur est $60 \%, 76 \%$ à
Bamako et $81 \%$ parmi les plus instruites. Au Mali $75,2 \%$ des femmes conviennent que le mari peut battre sa femme ${ }^{v}$ et seulement 27,3 $\%$ déclarent qu'une femme peut refuser des rapports sexuels lorsqu'elle est fatiguée ou pas d'humeur, alors que, pour le Ghana, ces chiffres sont de $36,6 \%$ et $82,5 \%$.

\section{Un projet de recherche multidiscip-} linaire et multiculturel appliquant l'Approche des Capabilités à des analyses secondaires de données existantes

Le projet a été élaboré et mis en œuvre par un consortium composé de l'Institut d'études démographiques et du parcours de vie (IDEMO), Université de Genève, et du Harvard Center for Population and Development Studies (Cambridge, USA), leurs partenaires à Bamako (Faculté de médecine, Université du Mali et la Direction nationale de la population) 
et à Accra (Département de géographie, Ghana School of Public Health et Institut régional d'études démographiques de I'Université du Ghana), avec la collaboration du Département de sciences politiques et sociales de l'Université de Pavie (Italie) (encadré I)

\section{Encadré I.}

Equipes de recherche. Projet 'Développer les Capabilités des plus vulnérables en Afrique de l'Ouest'.

Bamako : Prof. Samba Diop; Dr Sory Ibrahima Diawara, Samba Diarra, Dpt de santé publique, Faculté Médecine / Contribution de la Direction Nationale de la Population, Ministère économie.

Accra : Profs. Samuel Agyei-Mensah \& John K. Anarfi; Adu O.Sarkodie, Thomas Antwi Bosiakoh et Faustina Frempong (à Southampton), Dpt Geography \& Regional Institute Population Studies, University of Ghana.

Genève : Prof Cl. Sauvain-Dugerdil ; Mathias Lerch, Ababacar Dieng \& Anouk Piraut; I-DEMO, Université de Genève.

Harvard: Prof Allan G.Hill (actuellement à l'Université de Southampton); Nelly Douptcheva (à Geneva), Harvard School of Public Health.

Pavia : Prof. Enrica Chiappero-Martinetti: Nadia Von Jakobi \& Lia Quartapelle, Dpt of Political and Social Science, University of Pavia and the Human Development, Capability and Poverty International Research Centre, Institute for Advanced Study, Pavia (Italy)

Deux spécialistes des Capabilités ont rejoint l'équipe pour contribuer aux événements organisés par le projet: Prof. Sridhar Venkatapuram, King's College London, lors de la Table Ronde organise à la Conférence de l'Union internationale pour l'étude scientifique de la population (UIESP) à Busan en août 20I3, et Dr. Kevin Lompo, UMI-Résiliences de l'IRD Paris-Nord, à l'atelier de Bamako workshop en décembre 2013.

L'objectif principal du projet est d'appliquer l'AC en tant que nouvelle approche conceptuelle et méthodologique pour l'analyse de l'évolution des comportements démographiques et de leurs conséquences en termes d'inégalités de bien-être. II s'agit de déterminer si le cadre des Capabilités peut conduire à une exploitation plus complète des données existantes. Le projet a donc développé une méthodologie pour l'analyse secondaire de données d'enquête qui n'ont pas été recueillies dans la perspective de l'AC. Les analyses ont été menées sur deux types de données. D'une part, nous utilisons le riche matériel recueilli par les initiateurs du projet, c'est-à-dire l'enquête " Chantier Jeunes » sur la transition à l'âge adulte au Mali (voir les articles 4 et 7 ) et une enquête à grande échelle menée par les équipes de recherche sur la santé des femmes à Accra (article 3). D'autre part, nous avons effectué des analyses secondaires des grandes enquêtes nationales, notamment l'enquête sur les niveaux de vie au Ghana (article 6) et, dans les deux pays, l'enquête démographique et de santé la plus récente au moment de la réalisation du projet (articles 5, 8 et 9). En outre, des enquêtes qualitatives ad hoc ont été réalisées pour explorer certains facteurs associés aux notions de choix et de préférence (articles 5 et 10).

Appliquer I'AC implique un changement majeur par rapport à l'analyse classique des déterminants des réalisations (Ici les niveaux de fécondité, d'occupation, de morbidité et de prévalence contraceptive) pour mettre l'accent sur l'éventail des opportunités et les inégalités à 
cet égard, c'est-à-dire les "libertés des gens à être et faire ce qu'ils ont raison de valoriser". Nous avons choisi de travailler sur sept sujets dans trois domaines majeurs en démographie sociale, particulièrement pertinents en Afrique de l'Ouest, et pour lesquels de riches ensembles de données sont disponibles :

- La santé vue sous l'angle des facteurs de risque et de la montée de l'obésité;

- L'intégration sociale à travers l'activité professionnelle et la participation sociale;

- La planification familiale à travers la contraception et l'espacement des naissances.

Ces trois domaines font appel à une série de Capabilités figurant sur la liste de Nussbaum (2000) :

- La plupart des aspects qu'elle inclut dans la notion de santé corporelle, c'est-à-dire " être en mesure d'avoir une bonne santé, y compris en santé reproductive et de se nourrir convenablement ".

D'autres dimensions sont couvertes par ce que Nussbaum qualifie de:

- Sens, imagination et pensée: "être capable d'utiliser ses sens, d'imaginer, de penser et de raisonner - et de faire ces choses d'une manière 'véritablement humaine', une façon informée et cultivée par une formation adéquate [...]. Etre capable d'utiliser son imagination et sa pensée dans le cadre de ses expériences, de la production d'œuvres et d'événements de son choix $[. .]$.

- Affiliation : " être capable de vivre avec et vers les autres, [...] de s'engager dans diverses formes d'interaction sociale ".

- Raisons pratiques qui s'adressent aux questions de l'action individuelle (agency), exprimées par «la capacité à se former une conception du bien et à s'engager dans une réflexion critique sur la planification de sa vie ".

Chaque étude a élaboré un cadre analytique dans la perspective des Capabilités, mais spécifique au contexte et aux questions traitées. Toutes se réfèrent à un schéma de base commun (Figure I), ainsi qu'à des définitions communes (voir article précédant). Chaque étude a donc conceptualisé le duo Capabilités/Réalisations - c'est-à-dire en distinguant l'éventail de possibilités des réalisations réelles - ainsi que les différentes sources d'inégalités et a identifié les meilleurs variables, ou indicateurs composites, disponibles dans les données utilisées (cartographie des variable).

Figure I. Le cadre des Capabilités

(adapté de Robeyns 2005 vi et Chiappero \& Venkatapuram dans ce volume)

Ressources / dotation

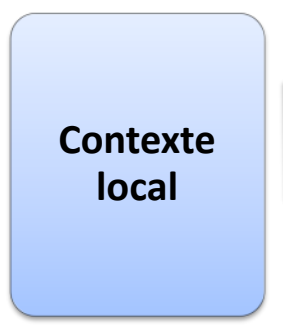

Capabilités

Fonctionnement
Préférences / Contraintes
Situation réelle des gens : Etre et Faire 
L'application de I'AC implique donc une stratégie en deux volets:
a) considérer les Capabilités comme une perspective pour choisir les meilleurs indicateurs du bien-être en termes de processus
b) identifier les différentes sources d'inégalités en matière d'opportunité et de réalisation.

\section{A. Capabilités et réalisations : la nature multidimensionnelle de la qualité de vie du point de vue de la personne.}

En considérant les réalisations comme les résultats sélectionnés parmi une gamme de possibilités réelles, l'AC se concentre sur le processus de création du bien-être. L'AC contribue donc au débat ancien sur la définition et la mesure de la qualité de vie d'au moins deux façons. D'une part, la distinction entre les possibilités et le résultat fournit un cadre pour saisir la nature multidimensionnelle du bien-être et de ses déterminants. D'autre part, le rôle de l'action individuelle occupe une place centrale qui implique la prise en compte d'indicateurs subjectifs qui traduisent le point de vue de l'individu et des informations sur les facteurs d'inégalité dans les aptitudes individuelles à accéder et utiliser les ressources disponibles.

Le principal défi dans l'application de I'AC à l'analyse démographique est d'identifier les variables qui peuvent être utilisés pour décrire adéquatement les Capabilités. Par définition, l'espace d'opportunités ne peut être pleinement mesuré. Les informations disponibles ne fourniront jamais une description exhaustive de toute la gamme des possibilités. De plus, elles se modifient avec le temps et la "liberté de les réaliser » se manifeste à travers la perception de la personne concernée. Les modèles économétriques introduisent les Capabilités par des variables latentes, ce que nous considérons peu satisfaisant car le plus souvent leur signification exacte est difficile à interpréter. Dans le présent projet, chaque étude de cas a identifié parmi les variables disponibles les meilleures indices pour quantifier le bien-être ici en matière de santé, d'intégration sociale et de planification familiale - en termes de capacité à être bien et à bien faire dans ces domaines de la vie, et les raisons pour lesquelles des personnes jouissant apparemment des mêmes conditions se retrouvent avec des situations différentes.

\section{* La « capacité de jouir d'une bonne}

santé " a été l'objet d'une enquête sur la santé auto-déclarée auprès des femmes d'Accra et de jeunes à Bamako. Dans l'étude d'Accra, les Capabilités sont considérées par la dispersion des indicateurs de santé dans cette population. Dans le cas de Bamako, nous testons si l'utilisation des services de santé constitue une Capabilité, c'est-à-dire si ces jeunes sont «effectivement en mesure d'accéder aux soins de santé de qualité» avec l'objectif d'améliorer leur état de santé. En ce qui concerne l'obésité, le cadre des Capabilités offre une façon fort pertinente pour démêler les différents types de facteurs de risques, en utilisant une méthodologie mixte quantitative-qualitative afin de dépasser les habituelle approches centrées sur la santé pour comprendre la signification que les gens donnent à l'obésité dans les contextes maliens et ghanéens.

\section{* La participation sociale et l'activité} professionnelle sont considérées comme des Capabilités en matière d'intégration sociale dans le sens de la capacité à utiliser les connaissances, les compétences et les réseaux pour «vivre la vie que l'on a des raisons de valoriser». En d'autres termes, nous considérons le contrôle qu'exerce la personne sur sa vie à travers le rôle que joue la formation pour ouvrir la voie à une occupation sûre. Deux autres études examinent le rôle de la participation sociale, d'une part, sur la conciliation de l'emploi féminin avec une famille nombreuse et, d'autre part, sur la liberté individuelle exprimée par le temps non structuré.

* L'étude "Dessiner sa famille» offre une alternative aux analyses classiques des déterminants de la fécondité et aux nombreux 
points de vue simplistes d'un lien de causalité entre l'utilisation de la contraception et la taille de la famille réalisée. Par une analyse secondaire des données EDS et une enquête qualitative, nous explorons la capacité des femmes à «avoir une bonne santé reproductive», c'est à dire les raisons pour lesquelles certaines femmes sont en mesure de gérer la constitution de leur famille et d'autres pas. Nous procédons en deux étapes. Tout d'abord, nous examinons si la connaissance de la contraception ouvre la possibilité de contrôler la fécondité grâce à l'utilisation de la contraception. Dans une deuxième étape, nous vérifions si les personnes qui adoptent la contraception moderne sont en mesure de planifier leur famille de façon efficace, tel qu'exprimé par l'espacement des naissances. Les données qualitatives sont utilisées pour examiner les rôles respectifs des normes culturelles, en particulier les anciennes traditions d'espacement des naissances et les nouveaux comportements plus individuels modulés par les différences de ressources personnelles.

\section{B. Les moyens de réaliser: différentes sources d'inégalité}

La capacité inégale de «vivre la vie qu'on a des raisons de valoriser" résulte de la disponibilité et de l'accessibilité de différents types de ressources, mais aussi de la capacité et de la volonté de les utiliser pour ses propres fins. L'AC strucuture l'analyse en faisant une distinction fondamentale entre les dotations et les dénommés facteurs de conversion. Elle fait en outre la distinction entre les ressources personnelles, familiales et sociales, ainsi qu'entre les ressources fixes et stables et celles qui évoluent avec les expériences de vie. Pour les questions démographiques, le cadre des Capabilités constitue donc un outil pour structurer l'analyse de l'hétérogénéité et revenir sur la distinction entre les facteurs de l'offre et de la demande.

Dans les études présentées ici, nous faisons usage des outils socio-démographiques habituels. Nous avons construit des modèles probabilistes (analyses de régression) pour examiner les facteurs de variabilité des indicateurs retenus pour définir les Capabilités, lesquels deviennent eux-mêmes, dans un second temps, des facteurs explicatifs de la réalisation. $\mathrm{Ce}$ choix a été fait comme alternative à une analyse de chemins, car il offre davantage de flexibilité dans l'examen de I'hétérogénéité. Globalement, nous avons procédé en testant d'abord l'effet des ressources du contexte et introduit ensuite les caractéristiques individuelles pour tester à la fois leur effet propre et si elles modifient l'effet du contexte. Le but est de faire ressortir les facteurs de vulnérabilité partagés par certains groupes de personnes, en particulier de distinguer le côté de l'offre - c'est à dire les ressources du contexte, notamment les services - du côté de la demande exprimé par les différences résultant des caractéristiques individuelles et du positionnement en terme de valeurs. L'enjeu principal est donc de savoir si les ressources personnelles peuvent modérer l'impact d'un environnement pauvre (voir en particulier article 3).

Les huit articles présentés ci-après illustrent bien que I'AC fournit une orientation globale pour la formulation de la question de recherche et pour structurer l'analyse, mais que sa mise en œuvre précise et l'identification des variables proxy dépendent de la question étudiée et, lors d'analyses secondaires, des informations disponibles.

Les ressources du contexte sont considérées comme substituts pour les dotations collectives. Dans le contexte malien et ghanéen, le lieu de résidence (urbain ou rural, type de secteur de la ville) est une indication valable sur les ressources disponibles et le degré de salubrité et peut donc être utilisé comme un proxy, mais bien sûr, il est préférable d'avoir des informations plus précises sur les conditions de vie réelle et les services tel que présentés dans certaines études (voir en particulier l'article 8).

En ce qui concerne les caractéristiques individuelles - parmi lesquels nous incluons également celles du ménage et de 
l'appartenance socioculturelle - le cadre des Capabilités distingue les ressources personnelles acquises de dotations fixes, c'est à dire les ressources personnelles qui peuvent être améliorées de celles qui ne peuvent pas être modifiées. Les conséquences qu'ont ces dernières sur le bien-être peuvent toutefois être modulées par les caractéristiques du contexte et leur signification sociale. Tel est le cas de deux variables centrales en démographie, l'âge et le sexe, qui constituent des dotations individuelles fondamentales porteuses d'inégalités à travers leur signification socioculturelle spécifique et leurs conséquences sur le pouvoir d'action de l'individu. Lorsque les informations sont disponibles, les analyses présentées dans ce volume prennent également en compte les spécificités associées à la situation dans le parcours de vie, en particulier les transitions telles que la parentalité ou l'emploi, ou l'histoire de vie par exemple l'effet des antécédents de santé (articles 3 et 4 ).

Dans toutes les études, une question centrale est celle des rôles respectifs des ressources humaines et économiques. Lorsqu'elles sont disponibles, les informations sur les infrastructures scolaires dans le secteur de résidence sont prises en compte comme une ressource de l'environnement, tandis que dans l'article 7 le niveau de scolarisation est considéré comme une dotation individuelle et dans les autres études, son effet net, après avoir contrôlé le niveau économique, comme un moyen d'utiliser les ressources (facteur de conversion). La richesse des ménages - une information disponible dans toutes les bases de données utilisés ici - est interprétée dans les articles des collègues économistes et de santé publique comme une ressource, alors que l'analyse socio-démographique la considère plutôt comme un moyen pour accéder et utiliser les ressources du contexte ${ }^{\text {vii. Dans les }}$ différentes études du présent projet, l'activité professionnelle est considérée soit comme un accomplissement - c'est-à-dire le type d'occupation et la sécurité de l'emploi comme indicateurs de la qualité de vie - soit comme une
Capabilité, un indicateur de la capacité de contrôle sur sa vie, mais aussi comme un facteur de conversion, à savoir un moyen pour atteindre d'autres facettes de bien-être.

\section{Une publication en trois sections}

Le but principal de ce Supplément est donc de fournir des illustrations de l'application de l'AC à une analyse secondaire de données qui n'ont pas été collectées dans la perspective des Capabilités. Par sept études dans trois domaines utilisant différents types de données réalisées par des chercheurs de différentes disciplines et institutions, il présente un échantillon de la diversité des possibilités d'application de l'AC dans les analyses sociodémographiques. Ces études sont mises en perspective par la présente section introductive et une dernière section, article de conclusion, qui résume les principaux résultats et les leçons à tirer du projet. II examine les forces et les faiblesses de l'AC, sa contribution aux études de population et aussi comment à l'avenir les analyses socio-démographiques pourraient dépasser certaines de ses faiblesses. Nous revenons aussi sur le défi de la prise en compte des inégalités dans la recherche scientifique et dans les politiques et programmes en discutant des thématiques qui nécessitent des développements pour mieux comprendre les sources d'inégalités et identifier les meilleurs indicateurs pour suivre et évaluer les actions.

\section{Références}

Bassand, Michel, Jean Kellerhals et Geneviève Wirth (1975), Familles urbaines et fécondité. Librairie de l'Université de Genéve.

Basu, K. (2013), Group identity, productivity and well-being: policy implications for promoting development. J Human Devel and Capabilities 14(3), 323-40.

Bhutta, Z. A. and R. E. Black (20|3), Global maternal, newborn, and child health-so near and yet so far. N Engl J Med 369 (23), 222635. 
Billari, Francesco and Alexia FürnkranzPrskawetz (2003),

Agent-Based

Computational Demography, Physica Verlag. Billari, Francesco, Dimitar Philipov and Maria R.

Testa (2005), The influence of attitudes, subjective norms and perceived behavioural control on union formation intentions. IUSSP Conference Tours (France).

Bonvalet, Catherine et Eva Lelièvre (dir. 20I2), De la famille à l'entourage. L'enquête biographies et entourage, INED, coll. " Grandes Enquêtes ».

Courgeau, Daniel (2007), Multilevel Synthesis. From the Group to the Individual. The Springer Series on Demographic Methods and Population Analysis, Vol. 18, 227 p.

Drèze, Jean and Amartya Sen (2013), An uncertain Glory. India and its contradictions. Allen Lane, Penguin Book, London.

Emirbayer, Mustafa and Ann Mische (1998), What is Agency? AJS I03(4): 962-1023.

Lesthaeghe, Ron (1995), The second demographic transition in western countries: an interpretation. In K. Oppenheim Mason and A.-M. Jensen: Gender and Family Change in Industrialized Countries, Oxford University Press, 17-62.

Lindenberg, Siegwart (1996), Continuities in the theory of social production function. In H.Ganzeboom and S.Lindenberg (eds): Verklarende Sociologie: Opstellen voor R. Whiple. Thela, Amsterdam.

Locoh, Thérèse et Zahia Ouadah-Bedidi (to be published), Du vin nouveau dans la vieille outre familiale: les Africaines en quête de nouveaux rapports de genre. Cahiers Québecois de démographie 2014.

Nussbaum, Martha (2000), Women and Human Development, Cambridge University Press, Cambridge.

Robeyns, Ingrid (2005), The Capability Approach - A theoretical survey, Journal of Human Development, vol 6, no I, 93-II4.

Sen, Amartya (1999), Development as Freedom. New York: Knopf Press.

Tabutin, Dominique, Catherine Gourbin et G. Beninguisse (2007), Surmortalité et santé des petites filles en Afrique. Tendances des années 1970 aux années 1990. Chap 5 in Locoh (ed): Genre et sociétés en Afrique. Cahier INED 160.

United Nations (2013), The Millennium Development Goals Report 2013. New York.

Van de Kaa, D. J. (1994), The Second Demographic Transition Revisited: Theories ancd Expectations. Pp. 8I-I26 in: G. C. N. Beets et al. (eds.). Population and Family in the Low Countries Lisse, Zwets and Zeitlinger.

Waage, Jeff et al (20/0), The MDGs : a crosssectoral analysis and principals for goal setting after 2015. The Lancet \& London Internat Dev Centre Commission. The Lancet 376, 991-1023.

Wilkinson, Richard and Kate Pickett (2009), The Spirit Level: Why More Equal Societies Almost Always Do Better. Allen Lane.

Woods, R. (2000), The demography of Victorian England and Wales. Cambridge ; New York: Cambridge University Press.

Woods, R. (2003), Urban-rural mortality differentials: An unresolved debate. Population and Development Review 29 (I), 29-.

Yamin, Alicia Ely and Vanessa M Boulanger (20I3), Embedding sexual and reproductive health and rights in a transformational development framework: lessons learned from the MDG targets and indicators. Reproductive Health Matters 2I (42):74-85.

' L'auteure remercie Allan Hill pour ses suggestions pertinentes et son travail d'édition, ainsi que les commentaires bien utiles d'Enrica ChiapperoMartinetti et de Shridar Venkatapuram sur une version antérieure.

ii Par exemple, dans les hautes vallées alpines de Suisse, la notion d'indigents est apparue comme une nouvelle catégorie au moment de l'arrivée du train au début du XXè siècle. 
http://www.unece.org/fileadmin/DAM/pau/icpd/C onference/Other documents/Report ICPD beyon d 2014.pdf

iv L'Indice de pauvreté multi-dimensionnelle (MPI) prend en compte le cumul de privations dans les trois mêmes domaines que l'IDH ; il exprime le nombre de personnes qui souffrent de pauvreté multidimensionnelle (privations dans au moins $33 \%$ des indicateurs pondérés) (Human

Development Report 2013, http://hdr.undp.org/en/statistics/mpi)

${ }^{\vee}$ Femmes qui approuvent qu'un mari batte sa femme sous certaines conditions: la femme brûle la nourriture, la femme argumente avec lui, sort sans lui dire, néglige les enfants ou refuse d'avoir des relations sexuelles avec lui.

vi Comme le souligne Robeyns (2005), ce schema ne doit pas être compris comme représentation dynamique. II ne figure pas des relations causales mais la direction des conditionalités : les facteurs de conversion sont contingent des ressources du contexte et les réalisations des Capabilités.

vii De façon similaire, I'utilisation de la contraception est considérée par les premiers comme une ressource et par les derniers comme un moyen. 